This item was submitted to Loughborough's Research Repository by the author.

Items in Figshare are protected by copyright, with all rights reserved, unless otherwise indicated.

\title{
Framework for a technology-watch relay station
}

PLEASE CITE THE PUBLISHED VERSION

PUBLISHER

(c) 2004 IP Publishing Ltd.

VERSION

VoR (Version of Record)

LICENCE

CC BY-NC-ND 4.0

REPOSITORY RECORD

Stokes, Chris A., and Paul J. Palmer. 2019. "Framework for a Technology-watch Relay Station”. figshare. https://hdl.handle.net/2134/6246. 
This item was submitted to Loughborough's Institutional Repository (https://dspace.lboro.ac.uk/) by the author and is made available under the following Creative Commons Licence conditions.

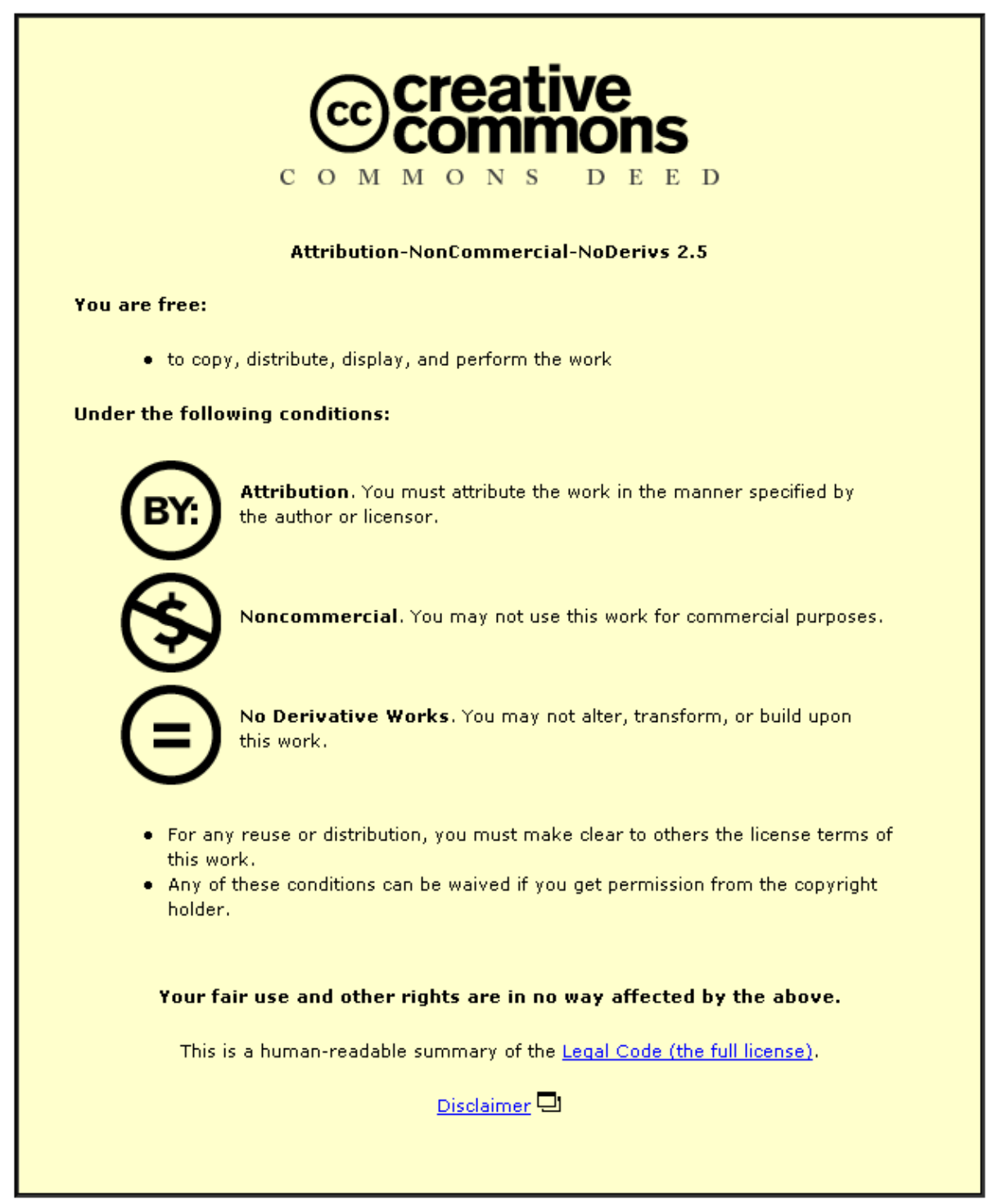

For the full text of this licence, please go to: http://creativecommons.org/licenses/by-nc-nd/2.5/ 


\title{
Framework for a technology-watch relay station
}

\author{
C. A. Stokes and P. J. Palmer
}

\begin{abstract}
At the centre of the PRIME Faraday Partnership's Technology Watch service is a growing series of technology and market reviews for managers and engineers in small and medium-sized enterprises (SMEs) producing 'smart' products. Its aim is to help them maintain their awareness of new technologies and markets and thereby seize opportunities to innovate they might otherwise miss. The service has attracted considerable interest among users and observers. In this paper the authors outline the process behind the development of Technology Watch's library of publications with a view to encouraging others to critique and adapt it for deployment in other industrial sectors.
\end{abstract}

Keywords: Technology Watch; technical intelligence; smart products; manufacturing; SMEs; innovation

The authors are with the PRIME Faraday Partnership, c/o Wolfson School of Mechanical and Manufacturing Engineering, Loughborough University, Loughborough, Leicestershire LE11 3TU, UK. Tel: +44 1509 227671. Fax: +44 1509227671.

E-mail: c.a.stokes@Iboro.ac.uk.

By maintaining an awareness of its environment, a company is best prepared to act effectively in that environment, whether to exploit emerging opportunities or to protect itself against emerging threats such as those posed, for example, by disruptive technologies (Bower and Christensen, 1995). This is especially critical for companies that manufacture 'smart' products, for these companies commonly find themselves in environments in which change is the rule.

As large original equipment manufacturers (OEMs) continue to divest themselves of all but their core competences (Kakabadse and Kakabadse, 2000; Morgan Chambers, 2001), small manufacturing companies are proliferating in their supply chains. The concomitant fragmentation of supply chains endangers their collective awareness of the environment in which they operate. Small suppliers are often so strongly focused on their day-to-day operations that looking beyond their suppliers and customers to scan the horizon for technological developments is a low priority.

Technology Watch aims to play a part in redressing the balance by making it easier for smart-product manufacturers to sensitize themselves to signs in their environment that presage new threats or opportunities. The principal means for this is the ongoing publication of a growing library of reviews of new product and process technologies, new markets, new techniques, new standards and regulations and other factors relevant to the target users' interests. Supported by the UK Department of Trade and Industry (DTI), this experimental service is provided by the PRIME Faraday Partnership (PRIME Faraday Partnership, 2004). Established among the first four Faraday Partnerships in 1997 (Quotec, 2004), this is the 
partnership that serves manufacturers of products with interdependent mechanical and electronic (PRIME) parts (and usually a computing part too). The partners are Loughborough University, the University of Nottingham and Pera, a company combining strengths in engineering $R \& D$, industry research and business support. The Stuttgart-based Fraunhofer TEG (Fraunhofer-Technologie-Entwicklungsgruppe) is an associate partner.

\section{Technology watch in general}

We deliberately state that Technology Watch aims to play a part in redressing the balance. That is to say, it does not offer a complete corporate 'technology watch' service in the usual sense of the phrase. Technology watch and its close relatives, known variously as 'technology intelligence' or 'competitor technology intelligence' (Lichtenthaler, 2003), 'technical intelligence' or 'competitive technical intelligence' and, in French-speaking countries, 'la veille technologique', all find their natural home in the large organization. When fully implemented in such a setting, technology watch provides a firm's executive team with timely intelligence tailored to its particular needs in the context of its own particular corporate strategy in its own particular environment. There are third-party specialist technology-watch service providers, it is true, but the service they provide is bespoke and confidential. It takes into account the specific goals and characteristics of the client company and its specific competitive environment. It is especially careful to take account of the technological interests of the company's competitors. It begins with the identification and collection of pertinent data and proceeds through data analysis to the dissemination of the resulting practical intelligence within the organization (Albagli, 1997; Davidson, 2001; Libutti, 2000; Coburn, 1999;

Quazzotti et al, 1999).

The PRIME Faraday Partnership's Technology Watch differs from this approach in several important respects. It is not bespoke, but aims rather to serve manufacturing companies collectively right across the broad PRIME spectrum. It publishes its reviews openly, one-to-many. Its selection and treatment of review topics is not biased by the strategy of any actual or potential user companies. It can, therefore, satisfy only a part of any user company's corporate technologywatch requirement. And the part that it aims to fulfil is what might be called the early 'pre-competitive' stage of the larger integrated technology-watch task - a relatively low-resolution scanning of the horizon that many PRIME companies would each carry out anyhow if they were to fully implement their own corporate technology watches. In Davidson's terms, the PRIME Faraday's Technology Watch is a kind of technologywatch 'relay station' (Davidson, 2001).

Bespoke commercial research and information services are commonplace. However, as far as the authors are aware, there is little experience of providing a service of the relay-station kind. Experience of the service to date suggests that the Partnership's Technology Watch, while admittedly still inchoate, offers a promising model for helping British PRIME manufacturing SMEs to keep abreast of important technology and market developments. Having in mind the possibility that it may be adapted for use in other industrial sectors or communities of interest, we outline here its modus operandi. This paper describes how the necessary elements were articulated into a framework for the Technology Watch operation and highlights some unforeseen obstacles that were encountered and how they were negotiated. Description of the elements themselves - where we find and how we collect and analyse our data - we must leave for another occasion.

The elements of Technology Watch discussed in this paper constitute an iterative cycle - a cycle in which new titles are selected, commissioned, researched and written and their appeal to the target audience is assessed before the next cycle begins (Figure 1). We do not consider here other elements of the Partnership's Technology Watch, such as the provision of a specialist real-time news service, an events diary and so on.

\section{Prime Faraday Technology Watch}

The PRIME Faraday Partnership's Technology Watch is a technology observatory, providing the kind of information needed by small and medium-sized PRIME manufacturers to take a strategic view of technologies. New technologies are of most interest. These may be experimental technologies (such as many nanotechnologies), but more usually they are technologies that are becoming widely available or became so no more than a few years ago (for example, MEMS, e-marketplace tools, virtual-prototyping systems, moulded interconnect devices), traditional technologies in new advanced forms (for example, many machining technologies), and technologies that have been in use for some time in other manufacturing sectors or large PRIME companies but not in many PRIME manufacturing SMEs (such as customer relationship management systems). All these technologies are currently exerting a new and material influence on the environment of PRIME manufacturers or may well do so over the next few years. The technologies that most obviously fit this bill are those that a Technology Watch user might consider 
incorporating in future products or future manufacturing processes, but they also include technologies and techniques that are important to manufacturing management and business administration.

The intended audience for a Technology Watch review is broader than the pool of experts in the subject under consideration. In fact, these are the secondary audience members: the primary targets are managers in PRIME manufacturing SMEs who may not have the same specialist knowledge of the review subject as, say, their design or R\&D engineers. The intended audience also includes specialists who are not expert in the subject under consideration.

A Technology Watch title does not deal narrowly with a technology. Those reviews with titles announcing that their subject is a technology (for example, Moulded Interconnect Devices) do more than summarize the functions, applications and physical principles of that technology. Ideally, they draw together engineering and scientific aspects of the technology and other issues pertinent to its future potential, and these may be legal, social, demographic, economic, financial, industrial, psychological, perhaps even aesthetic issues.

Many reviews take topics other than technologies as their main theme - among them industrial regulations (for example, environmental legislation) and management tools and techniques (for improving productivity, developing new products and marketing technology products, etc). But again the ideal is to avoid a narrow focus: Technology Watch reviews should give due consideration to the heterogeneity of factors relating to the decision on how to respond to a new technology. The reviews refuse to oversimplify, to pretend that the decision can be made effectively by filtering out all but the technological and scientific aspects, to take for granted the myth of technological determinism (Griffith and Dougherty, 2001) that technologies evolve according to a hard technological (and therefore asocial) logic (Latour, 1987; Law, 1987).

\section{The Technology Watch process}

The remainder of this article describes the iterative cycle by which Technology Watch's library of reviews is continually expanded. For the sake of clarity, we present the cycle as a sequence of discrete steps in which the last loops back to the first to close the circle (Figure 1). In reality, of course, the cycle is not so simple: there are sub-cycles inside the larger cycle, for example. Moreover, what are here represented as discrete steps do in fact blur at the edges into what is in reality a much more fluid process. We begin with a planning step, the step that results in a schedule of new titles to

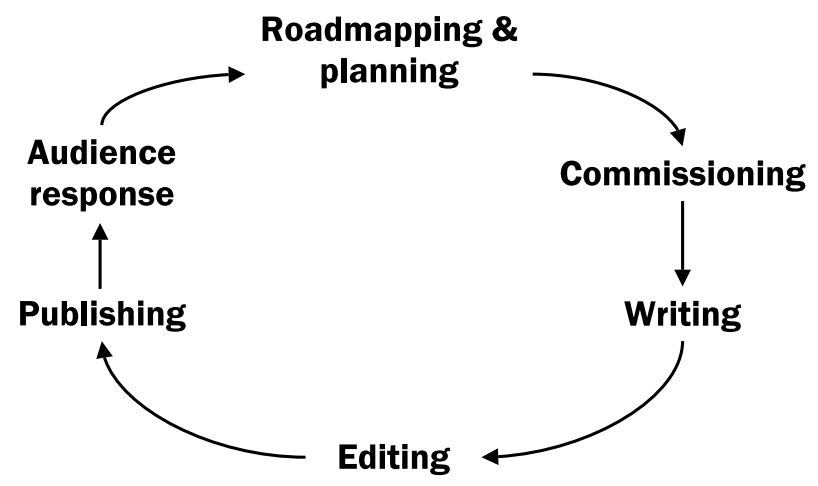

Figure 1. The Technology Watch process.

be researched, written and added as new publications to the Technology Watch collection of reviews.

\section{Planning}

The planning step is characterized by one (or occasionally more) face-to-face meetings in which Technology Watch representatives from each of the Partnership's three main partner organizations - Pera, Loughborough University and the University of Nottingham - brainstorm and then discuss the range of options generated, winnowing it down to the number of titles that can be accommodated by the Technology Watch budget. Rejected titles that just miss inclusion are carried over to the next planning meeting. Factors taken into consideration are many and varied, but typically include the relative popularity among Technology Watch users of subjects already reviewed. The meetings are informed by an evolving PRIME technology roadmap, which emanates from continuing parallel research on technology roadmapping led by one of the authors of this paper (PJP). Informal unwritten guidelines further limit the freedom of choice at the planning stage: for example, we aim overall to cover the PRIME domain more or less evenly and thus to avoid a bias towards any preordained area within it; we similarly aim to avoid large overlaps in the subject areas treated by different reviews.

A new list of future titles cannot be formulated without reference to resource constraints. It is no accident that Pera, with its proven experience in researching and collating reports on technologies and markets, is one of the partners in the project. On the other hand, Technology Watch does not have the resources to seek out and commission for each review an author who can combine a high degree of domain expertise with the skill of writing engagingly for a nonspecialist audience. The majority of reviews published to date have been researched and written by Pera 
employees who, when necessary, make up for a lack of domain expertise through careful research. The remaining significant minority of Technology Watch reviews have been commissioned from external domain experts.

\section{Commissioning}

The leader of the Pera team of Technology Watch researcher-writers participates in the meetings at which future titles are brainstormed, proposed, discussed and assessed. This helps ensure inter alia that the team does not take on titles that it will be unable to produce to its high standards (it also helps to balance the workload across the team members). Titles that fall into this category and that the PRIME Faraday partners collectively judge to be indispensable to the Technology Watch library of reviews are the ones that are commissioned from external domain experts.

While no formal commissioning process is necessary for the reviews produced by Pera, for the 'outsourced' reviews, the Partnership needs to satisfy itself that the domain experts tentatively identified as authors are really appropriate. An important consideration is a potential author's ability and willingness to deliver a review of the required breadth, and this issue has to be raised early in the Partnership's discussions with the candidate. Experts with intimate technical engineering knowledge and experience of a subject are often not used to framing it in the broad terms required by Technology Watch.

Once a domain expert has informally agreed to write a review, a formal contract is drawn up and signed. A crucial ingredient in this contract is a clause in which the author assigns to the Partnership all rights in the final manuscript. Without such a clause, copyright would remain with the commissioned author and timeconsuming licensing complications would ensue for the Partnership.

\section{Writing and copyright}

The writing of a review is left largely to the author or authors. In terms of content, nothing more prescriptive is imposed than guidelines on the breadth of coverage (mentioned above), the target readership, the inclusion of graphics (along the lines of 'a [well-chosen] picture speaks a thousand words' and aids readability), and so on. Authors are also asked to follow a basic house style and to use a word-processor template specially designed for Technology Watch publications.

All authors also receive an in-house guide to copyright and the acknowledgement of sources. Highlighting the implications of copyright specific to Technology Watch, this document is required reading for review authors. It is no surprise that a detailed understanding of copyright (and the related sui generis database right) is to be found only among copyright lawyers - it is a highly complex area of law. Thanks to the proliferation of modern reproduction and publishing technologies, the much amended UK Copyright, Designs and Patents Act 1988 has the job of governing the now devilishly complex array of uses to which a text, graphic, moving image, sound recording or any multimedia combination of these can be put. While it would be unreasonable to expect present and future Technology Watch authors to be expert in the law, the Partnership cannot afford to publish reviews that infringe copyright - a copyright lawsuit could easily put a service on the scale of Technology Watch out of action.

\section{Editing}

A review written in-house normally requires writing just once. Before the writing proper begins, the writing team and the editor reach agreement on a brief outline of the contents of the review. The next version of the review that the editor sees is the complete first draft. Because of the Technology Watch culture that has developed among those who contribute to the programme, the first draft is made ready for publication in a single editing phase. Occasionally the editor may need to speak to the author - to clarify the meaning of an ambiguously worded passage, for example - but for most reviews nothing more is required in the editing phase than proofreading corrections and a small degree of style editing.

The editing of reviews commissioned from outside experts tends to be more complicated. The first brief outline of the proposed review is normally drafted by the editor after early consultation with the writer. The editor prefers to see an early draft of the review about half-way through the period agreed in the contract - if necessary, this draft can be incomplete. Much depends on how the author is accustomed to writing documents of the length typically sought (10,000-20,000 words) and also on how confident the editor and author are that they share a common understanding of what is to be written. At this stage, it is not unusual for the editor to request substantial changes in relation to style, content, organization, accessibility to non-specialists, etc. Later, it is not unusual for the editor to request extensive rewriting of the first complete draft. Needless to say, an editor will benefit from an ability to keep writers to deadlines.

\section{Publishing}

Until 2003, completed reviews were published solely on a Website dedicated to the Partnership's Technology Watch service (http://www.primetechnologywatch.org.uk/). 
Online publication has several advantages over print. In particular, it saves the publisher the expense of printing and warehousing an inventory of printed books and obviates the risk of losing money on stock for which orders may never materialize. In the case of Technology Watch, the wherewithal required has not stretched much beyond the following: the services of a reliable Internet service provider managing a dedicated server for the project; domain-name registration; the services of a Website design team (in-house); software for converting completed reviews as word-processor files into Adobe's portable document format (pdf); and software for analysing the log files that are generated by the Web server as a record of Website activity.

Publishing Technology Watch online has, however, brought a few of its own problems. One of these has been the difficulty of ensuring that someone who searches for information that happens to be contained in a Technology Watch review on the Web will find it. Search engines bring a sizeable proportion of all visitors to the Technology Watch Website $-26 \%$ at the time of writing (October 2004). In response to a search query, the more sophisticated search engines will include in the results a link to a pdf document when the search query matches text inside the pdf. Less sophisticated engines search no deeper than the HTML file in which the link to the pdf document appears this was the very best that could be expected a few years ago. Unfortunately, some sophisticated search engines drop links to some Technology Watch reviews (pdfs) from time to time, with a resulting large fall in the number of people accessing these reviews until the search engine reinstates the links. This problem arises from the continual change and growth of the Internet content that the search engines are attempting to index and the continual corresponding changes in searchengine indexes. There is no easy solution, because for obvious reasons search-engine providers do not reveal how their search engines work. The response frequently recommended by Internet commentators is to ensure that a document has as many third-party links as possible on other Web pages, especially on those pages already highly rated by search engines. The importance of public relations, therefore, is not to be underestimated.

In summer 2003, Technology Watch ventured into print publication for the first time when it issued the first thirteen of its reviews in paperback. It was hoped that by opening up this extra channel the reviews would be brought to the attention of a larger proportion of the target audience. The books are available on www.amazon.co.uk, but print publications are also catalogued by large bibliographical data services in the
UK, such as Whitaker and Nielsen Bookdata, whose online catalogues are widely used by booksellers and librarians.

The popularity of desktop publishing may lead people to believe that short-run print publishing is now straightforward (leaving aside the financial risks of holding stock). We were uninitiated and it proved not to be plain sailing. A tranche of ISBN numbers needed to be purchased - without them titles are not catalogued by the bibliographical data services and Amazon.co.uk will not accept them for its 'Advantage' scheme, under which it sells and delivers a publisher's titles and deals with the order processing in exchange for a percentage of the cover price. The book covers, and the internal layout of some reviews, were designed and redesigned respectively. A decision was taken for each title on whether to print digitally (for small runs) or conventionally (if the run was sufficiently large). Printing companies were approached and one was selected. It helped that this company was able to print from digital files in pdf format. Then suitable premises for storing the stock had to be found. And, of course, we had to publicize the new availability of Technology Watch reviews in print.

\section{Audience response}

The evolution of a service like Technology Watch, which is of necessity experimental, is determined to some extent by trial and error. For the service to survive or, better, become evermore useful to its users, there must be feedback mechanisms through which user responses can be channelled and considered, and a system that enables the lessons learned from such responses to inform modifications to the service.

At the time of writing, it must be admitted that the mechanisms of this kind for Technology Watch are not yet mature. The Website has a page for submitting written feedback directly, as well as one that lists postal and e-mail addresses for Technology Watch staff, but little feedback has been received to date via these routes. We currently learn about users and their views primarily through a software package for analysing Web-server log files, the contents of a database in which 'subscribing' users enter details about themselves (in exchange for access privileges) and informal channels such as conversations at meetings, seminars and conferences.

The log-analysis software is technically sophisticated, but its analyses are inevitably ambiguous because of the many possible behaviours concealed behind many of its statistics. For example, it is not possible to ascertain how many of the users recorded as having visited a particular review in a given month actually gleaned anything useful from it and how many 
quickly realized that it held no interest for them. The database of subscriber details lists a few details entered by users who have opted to supply them in exchange for instant access to newly published reviews (rather than wait for the end of an embargo period for nonsubscribers). There has not yet been enough time, however, for it to log a large number of subscribers and the information that can realistically be collected from each is limited. As for the informal channels, they can provide rich detail but they are unsystematic and sporadic.

In future, a much more interactive role is envisaged for Technology Watch - one in which information provider and audience work more closely together. Where possible, it is hoped that partnerships can be formed with alliances of opinion-leading manufacturers. This could greatly enhance our appreciation of the audience's response to the Technology Watch output and thus improve the match between what is provided and what the audience needs. Such alliances could also participate in other stages of the Partnership's Technology Watch process, notably in the planning stage. One long-term aim is to build a national community of interest linking smart manufacturers with one another and with other interested organizations, from universities to businesssupport organizations.

\section{A way ahead}

Since the PRIME Faraday Partnership's Technology Watch was launched, services of a similar kind have come on-stream, apparently with increasing frequency. Central sources - other Faraday Partnerships and some regional development authorities, for example disseminate information about new technologies to large audiences comprising many heterogeneous companies, mostly SMEs. We suspect nevertheless that this kind of service is comparatively untried at present, and that the knowledge of what works when and how is locked up in a few technology-watch relay stations that barely know of one another's existence. Despite our different foci, our collective chances of increasing innovation among our audience members might be much improved if we were to make a concerted effort to recognize one another and record and then communicate among ourselves the knowledge we have accumulated to date. We hope this article takes a step in that direction.

\section{References}

Albagli, A. (1997), 'Science and technology insights in SMEs', International Journal of Information Sciences for Decision Making, Vol 1, No 1, pp 19-27.

Bower, J. L., and Christensen, C. M. (1995), 'Disruptive technologies: catching the wave', Harvard Business Review, Vol 73, No 1, pp 43-53.

Coburn, M. M. (1999), Competitive Technical Intelligence: a Guide to Design, Analysis and Action, Oxford University Press, New York.

Davidson, C. H. (2001), 'Technology watch in the construction sector: why and how?', Building Research \& Information, Vol 29, No 3, pp 233-241.

Griffith, T. L., and Dougherty, D. J. (2001), 'Beyond sociotechnical systems: introduction to the special issue', Journal of Engineering and Technology Management, Vol 18, No 3-4, pp 207-218.

Kakabadse, N., and Kakabadse, A. (2000), 'Critical review outsourcing: a paradigm shift', Journal of Management Development, Vol 19, No 8, pp 670-728.

Latour, B. (1987), Science in Action: How to Follow Scientists and Engineers Through Society, Open University Press, Milton Keynes.

Law, J. (1987), 'Technology, closure and heterogeneous engineering: the case of the Portuguese expansion', in Bijker, W. E., Hughes, T. P., and Pinch, T. J. eds, The Social Construction of Technological Systems, New Directions in the Sociology and History of Technology, MIT Press, Cambridge, MA, pp 111-134.

Libutti, L. (2000), 'Building competitive skills in small and medium-sized enterprises through innovation management techniques: overview of an Italian experience', Journal of Information Science, Vol 26, No 6, pp 413-419.

Lichtenthaler, E. (2003), 'Third generation management of technology intelligence processes', R\&D Management, Vol 33, No 4, pp 363-751.

Morgan Chambers (2001), Outsourcing in the FTSE 100: The Definitive Study, Morgan Chambers, London.

PRIME Faraday Partnership (2004), 'PRIME Faraday Partnership', http://www.primefaraday.org.uk/, 11 November.

Quazzotti, S., Dubois, C., and Dou, H. (1999), Veille technologique: guide des bonnes pratiques en PME/PMI, Centre de Veille Technologique, Luxembourg.

Quotec (2004), 'Faraday Partnerships: wealth from knowledge', http://www.faradaypartnerships.co.uk/index.html, 11 November. 